Coarse grain modeling of spall failure in molecular crystals: role of intra-molecular degrees of freedom

This article has been downloaded from IOPscience. Please scroll down to see the full text article.

2009 Modelling Simul. Mater. Sci. Eng. 17015007

(http://iopscience.iop.org/0965-0393/17/1/015007)

View the table of contents for this issue, or go to the journal homepage for more

Download details:

IP Address: 128.46.221.4

The article was downloaded on 29/11/2010 at 17:21

Please note that terms and conditions apply. 


\title{
Coarse grain modeling of spall failure in molecular crystals: role of intra-molecular degrees of freedom
}

\author{
Karen Lynch $^{1}$, Alexander Thompson ${ }^{1}$ and Alejandro Strachan ${ }^{1,2,3}$ \\ ${ }^{1}$ School of Materials Engineering, Purdue University West Lafayette, IN 47907, USA \\ 2 Birck Nanotechnology Center, Purdue University West Lafayette, IN 47907, USA \\ E-mail: strachan@purdue.edu
}

Received 17 March 2008, in final form 3 November 2008

Published 25 November 2008

Online at stacks.iop.org/MSMSE/17/015007

\begin{abstract}
We use a recently developed thermodynamically accurate mesodynamical method (Strachan and Holian 2005 Phys. Rev. Lett. 94 014301) where groups of atoms are represented by mesoparticles to characterize the shock compression and dynamical failure (spall) of a model molecular crystal. We characterize how the temperature rise caused by the shockwave depends on the specific heat of the degrees of freedom (DoFs) internal to the mesoparticles $\left(C^{\text {int }}\right)$ and the strength of the coupling between the internal DoFs and the mesoparticles. We find that the temperature of the shocked material decreases with increasing $C^{\text {int }}$ and decreasing coupling and quantify these effects. Our simulations also show that the threshold for plastic deformation (the Hugoniot elastic limit) depends on the properties of the internal DoFs while the threshold for failure is very insensitive to them. These results have implications on the results of all-atom MD simulations, whose classical nature leads to a significant overestimation of the specific heat of molecular materials.
\end{abstract}

\section{Introduction}

A more fundamental and predictive understanding of the thermo-mechanical properties of complex molecular solids is critical in a wide range of areas from pharmaceuticals [1] to high energy density materials [2]. Classical molecular dynamics (MD) simulations can help fill this gap by providing detailed information about the molecular-level mechanisms that govern the thermo-mechanical response of materials and it has already provided invaluable information in areas such as shock-induced plasticity [3], dislocation structure and properties [4,5], size effects on deformation [6] and chemical reactions induced by mechanical stimuli [7]. However, two limitations hinder the widespread application of classical MD in the study of molecular materials: (i) all-atom simulations are very intensive computationally due to the high-frequency

3 Author to whom any correspondence should be addressed. 
vibrations associated with many intra-molecular degrees of freedom (DoFs) and the long range electrostatic interactions originating from atomic charges often present in these materials and (ii) an inaccurate description of thermal properties due to the quantum nature of the high-frequency, high-energy vibrations. While the first limitation is a technical one and the fast increase in computing power and the development of more efficient algorithms is alleviating its impact [8], the second one is of a fundamental nature. Classical MD is based on classical mechanics and it leads to classical statistical mechanics. This, in turn, leads to an inaccurate description of high-frequency roto-vibrational DOFs whose energy is larger than or comparable to the thermal energy available to the system $k T$ (where $k$ is Boltzmann's constant and $T$ represents temperature). A classical treatment of high frequency modes leads to an overestimation of the specific heat. Quantum effects on atomic dynamics can be approximately incorporated via methods based on Feyman's path integral formalism such as centroid dynamics [9]; however these methods are computationally more intensive than classical MD and remain restricted to relatively small systems. In this paper we focus on a different approach: coarse grain simulations, where mesoparticles represent groups of atoms, and a recent development enables the incorporation of the thermal role of atomic DoFs not explicitly [10] including the possibility of incorporating the quantum nature of high-frequency roto-vibrational modes. We use this new method, denoted dynamics with implicit degrees of freedom (DID), to characterize the role of the thermal properties of intra-molecular DoFs in the mechanical response of a model fcc molecular crystal when subjected to high deformation rates. We focus on plastic deformation and dynamical failure (spallation) caused by shock loading and the subsequent expansion and how these processes are affected by the specific heat of the implicit DoFs and their coupling with the mesoparticles.

Shock compression and dynamical failure are complex processes that involve a variety of phenomena such as (i) the nucleation and propagation of defects (often dislocations) during compression to release the uniaxial compression of the shock towards a more hydrostatic stress state, (ii) the formation and growth of voids and cracks during the tensile regime and (iii) the interaction and interplay between the various defects. An additional complication is that these processes occur under high stress and strain rates. Shock compression and dynamical failure provide important measures of the strength of materials and their deformation mechanisms under extreme conditions and consequently extensive experimental and theoretical efforts have been devoted to their study. While recent experimental advances such as ultra-fast x-ray [11, 12] and infrared spectroscopy [13] enable the characterization of dynamical loading with unprecedented resolution theory and simulation (see, for example $[14,15])$ continue to play an important role in the development of a physics-based, atomic-level understanding of high strain-rate phenomena that remain elusive, especially in molecularly complex materials. Thus, understanding the strengths and limitations of current simulation techniques is an important step for future developments in the field.

This paper is organized as follows. In section 2 we briefly describe the DID method, the model molecular crystal employed and the setup of the non-equilibrium simulations. In section 3 we present our results and section 4 discusses the implications of our work and draws conclusions.

\section{Simulation details}

\subsection{Dynamics with implicit DOFs for coarse grain modeling}

In mesoscale or coarse grain modeling, groups of atoms (an entire molecule in this paper) are represented by mesoparticles; these simulations are computationally less intensive than 
all-atom MD due to the reduction in the number of DoFs and the fact that high-frequency modes are not described explicitly enabling longer integration time-steps. Such mesoscale techniques are widely used in the simulation of soft materials where mesoparticles represent molecules [10] or groups of atoms in polymers [16,17]; additionally, a mesoparticle representation of polycrystalline materials was recently proposed by Holian [18].

In most approaches the thermodynamic state of the implicit DoFs (atomic positions measured from the mesoparticle's center of mass) is assumed to remain constant during the simulation. However, in reality the internal DoFs continuously exchange energy with the mesoparticles and neglecting this process leads to important inaccuracies. For example, if an electromagnetic field couples with a set of intra-molecular DoFs and increases their energy, this extra excitation will eventually be shared by all DoFs (including inter-molecular ones) according to the principles of quantum statistical mechanics. The DID equations of motion [10] enable such energy exchange describing the implicit DoFs via generalized, finite thermostats. DID describes the state of each particle (indexed with subscript $i$ ) with three variables: position $\left(r_{i}\right)$, velocity $\left(u_{i}\right)$ and internal temperature $\left(T_{i}^{\mathrm{int}}\right)$. This last variable describes the thermal state of the DoFs internal to the particle. The DID equations of motion are

$$
\begin{aligned}
& \dot{r}_{i}=u_{i}+v\left(\frac{T_{i}^{\text {meso }}-T_{i}^{\text {int }}}{\theta^{0}}\right) \frac{F_{i}}{m_{i}\langle\omega\rangle^{2}} \\
& \dot{u}_{i}=\frac{F_{i}}{m_{i}} \\
& \dot{E}_{i}^{\text {int }}=C_{i}^{\text {int }} \dot{T}_{i}^{\text {int }}=v\left(\frac{T_{i}^{\text {meso }}-T_{i}^{\text {int }}}{\theta^{0}}\right) \frac{F_{i} \cdot F_{i}}{m_{i}\langle\omega\rangle^{2}},
\end{aligned}
$$

where $F_{\mathrm{i}}$ and $m_{\mathrm{i}}$ denote the force and mass of particle $i$ and the dot denotes the time derivative. $C_{\mathrm{i}}^{\text {int }}$ is the specific heat associated with the internal DoF of mesoparticle $i,\left\langle\omega^{2}\right\rangle$ is the mean square frequency of the mesoparticles vibrational modes and $E_{i}^{\text {int }}$ is their internal energy corresponding to $T_{i}^{\text {int }}$. $T_{i}^{\text {meso }}$ is the local kinetic temperature of the mesoparticles in the neighborhood of particle $i, \theta^{0}$ is a reference temperature and $v$ (with unit of inverse time) represents the strength of the coupling between the internal and mesoscale DoFs [10]. The value of $v$ depends on the vibrational spectra of the individual molecules and how it couples with the vibrations of the solid. From the last of equations (1) we see that no energy flows between the mesoparticles and the internal DoFs if the local mesoparticle temperature is equal to that of the internal DoFs and energy flows from the DoFs with higher temperature to the colder ones.

These equations of motion lead to the conservation of linear momentum and total energy that consists of contributions from the mesoparticles and the internal DOFs:

$$
E^{\text {tot }}=E^{\text {meso }}+E^{\text {int }}
$$

where $E^{\text {meso }}$ is the sum of kinetic and potential energy of the mesoparticles:

$$
E^{\text {meso }}=\sum_{i} \frac{1}{2} m_{i} v_{i}^{2}+V\left(\left\{r_{i}\right\}\right),
$$

and the internal energy is given by

$$
E^{\mathrm{int}}=\sum_{i} E_{i}^{\mathrm{int}}\left(T_{i}^{\mathrm{int}}\right)
$$


(a) $\mathrm{t}=\mathbf{0} \mathrm{ps} \quad \mathrm{u}_{\mathrm{p}}=0.8 \mathrm{~km} / \mathrm{s}$

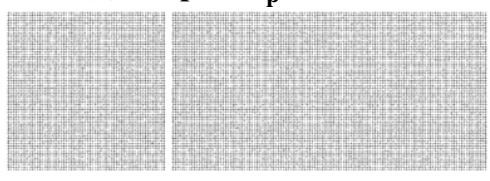

(b) $\mathrm{t}=5 \mathrm{ps}$

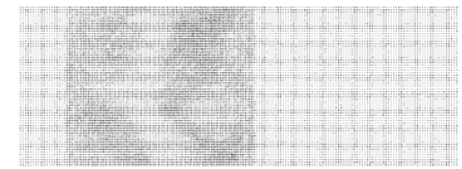

(c) $\mathbf{t}=\mathbf{1 7 . 5} \mathrm{ps}$

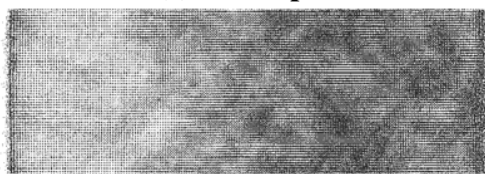

(d) $42.5 \mathrm{ps}$

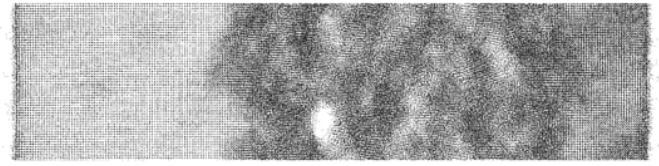

Figure 1. Atomic snapshots of a high impact velocity MD simulation at various times. The projectile consists of 8000 mesoparticles $(40 \times 40 \times 40 \mathrm{fcc}$ unit cells) and the target is composed of 16000 mesoparticles $(40 \times 40 \times 80 \mathrm{fcc}$ unit cells $)$. (a) Initial configuration. (b) Snapshot corresponding to $5 \mathrm{ps}$; compressive waves can be seen in the projectile and target. (c) At time $17.5 \mathrm{ps}$ when the compressive waves reach the free surfaces they are reflected back as tensile waves. ( $d$ ) At time $50 \mathrm{ps}$ failure of the material can be seen in the region where the two tensile waves meet.

\subsection{Interactions between mesoparticles: a model fcc molecular crystal}

We use the simplest possible description for a generic fcc molecular crystal and describe the molecular interactions with a pairwise mesopotential with the Morse form:

$$
E_{\text {Morse }}(r)=\varepsilon\left[\exp \left(\gamma\left(1-\frac{r}{r_{0}}\right)\right)-2 \exp \left(\frac{\gamma}{2}\left(1-\frac{r}{r_{0}}\right)\right)\right],
$$

where $\varepsilon=7.06 \mathrm{kcal} \mathrm{mol}^{-1}, \gamma=15.2$ and $r_{0}=7.1750 \AA$ and the molecular mass is $296.167 \mathrm{~g} \mathrm{~mol}^{-1}$. These parameters lead to a density of $1.84 \mathrm{~g} \mathrm{~cm}^{-3}$, a cohesive energy of $44.1 \mathrm{kcal} \mathrm{mol}^{-1}$ per molecule and a bulk modulus of $15.1 \mathrm{GPa}$; these values correspond to HMX [cyclic $\left[\mathrm{CH}_{2}-\mathrm{N}\left(\mathrm{NO}_{2}\right)\right]_{4}$ ], a high energy material used in propellant and explosive formulations. We emphasize that we simulate a model material that is not designed or expected to represent HMX beyond the three properties described above. A more truthful coarse grain representation of HMX would require a more complex mesopotential, see, for example [19], and possibly more than one mesoparticle per molecule.

\subsection{Non-equilibrium simulations of shock loading and spall}

We model dynamical loading and failure using high-velocity impact simulations of a projectile and a target both consisting of perfect crystalline samples of the molecular material, see figure 1(a) for a snapshot of the initial condition. In our setup the projectile contains 8000 
molecules and is obtained by replicating an fcc unit cell 40 times in the $x$ direction, 40 times along $y$ and 40 times along $z$ and the target contains 16000 molecules $(40 \times 40 \times 80$ unit cells); the target and projectile are initially separated by $1 \mathrm{~nm}$ along the shock direction $(z)$; see figure 1(a). Periodic boundary conditions are applied in the two directions normal to the shock propagation, along which the material is free to expand; the size and shape of the simulation cell are kept constant throughout the run. Such a setup [20,21] models a small region of a system consisting of two thin slabs. The simulated region is located far away for the free surfaces normal to the shock direction such that the relaxation of surfaces normal to the shock direction does not affect its dynamics since the timescales involved in the process of loading and failure (about $25 \mathrm{ps}$ in our case) are shorter than the time necessary for a sound wave to travel from the lateral surfaces to the region of interest. Thus, the cross-sectional area of the simulation cell is kept constant during our simulations.

After thermalization of the projectile-target system a non-equilibrium impact simulation is started by assigning a velocity of $+2 / 3 u_{\text {imp }}$ to all the molecules in the projectile on top of their thermal velocities and an extra velocity of $-1 / 3 u_{\text {imp }}$ to the target molecules (since the projectile is twice as massive as the piston the center of mass velocity of the whole system is zero). The temporal evolution of the mesoparticles is then solved using the DID equations (equations (1)) leading to a collision that launches two shockwaves (one into the projectile and one into the target) with piston velocities $u_{\mathrm{p}}=1 / 2 u_{\mathrm{imp}}$. The two waves and their shock fronts can be seen in the snapshot shown in figure $1(b)$ corresponding to $u_{\mathrm{p}}=0.8 \mathrm{~km} \mathrm{~s}^{-1}$. When the compressive waves reach the free surfaces the material expands into free space and tensile waves start propagating back into the sample; the snapshot shown in figure 1(c) shows one such tensile wave (denoted rarefaction fan) traveling into the projectile from the left while the shock wave in the projectile is reaching the free surface. Since the length of the target is twice that of the projectile the two rarefaction fans will meet roughly half-way into the target causing large tensile stresses and, for strong enough shocks, dynamical failure. This can be seen in the snapshot in figure $1(d)$ where several voids can be observed; in such high strain-rate regimes the percolation of voids that nucleate in the perfect materials leads to loss of cohesion and failure [20].

We characterized the response of the molecular crystal for a wide range of piston velocities $\left(0.2 \mathrm{~km} \mathrm{~s}^{-1}<u_{\mathrm{p}}<1.4 \mathrm{~km} \mathrm{~s}^{-1}\right)$ and different properties of the internal DoFs. We varied the specific heat of the internal DoFs from zero (corresponding to regular MD) to $80 k_{\mathrm{B}}$ (and $1000 k_{\mathrm{B}}$ in some simulations) and used two different coupling constants $v=0.042 \mathrm{ps}^{-1}$ (hereafter denoted strong coupling) and $v=0.0042 \mathrm{ps}^{-1}$ (weak coupling).

\subsection{Analysis of the mesoscale simulations}

In order to follow the process of shock loading and unloading we compute local properties (velocities, temperature and density) as a function of position along the shock direction at various times. To accomplish this, we divide our sample into thin slabs of thickness $3.448 \AA$ and compute the local properties of the molecules in each slab. The overall translation (center of mass velocity) is subtracted from molecular velocities to calculate local temperatures.

We also performed a detailed analysis of the DID trajectories to identify and quantify plastic deformation caused by the large deviatoric (non-hydrostatic) stress state caused by shock loading. This analysis is based on comparing the relative displacement of pairs of nearest-neighbor molecules at time zero (before any deformation has occurred) with that at the time of analysis. Such displacements enable us to identify pairs of molecules on either side of an active slip plane and quantify the Burgers vector of the dislocation involved. A more detailed description of the analysis method is given in [8]. 

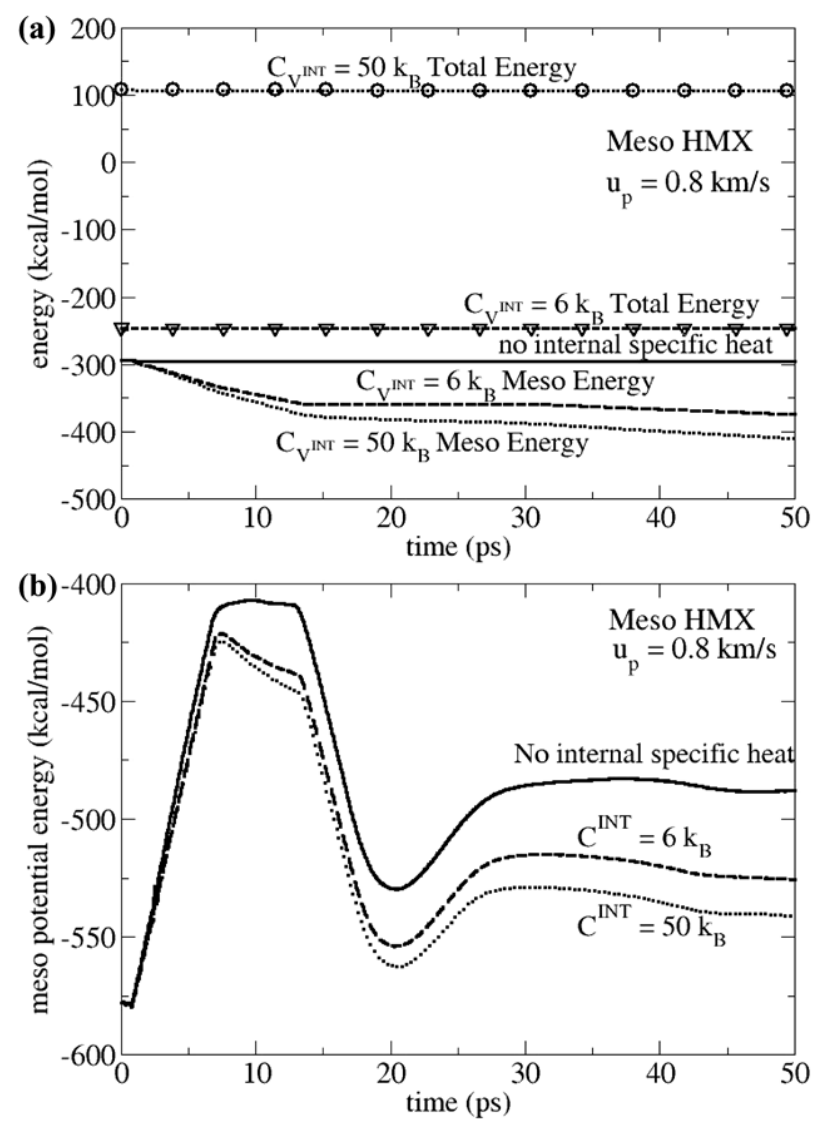

Figure 2. (a) Temporal evolution of total energy (internal + mesoparticles) and the energy of the mesoparticles for various internal DoFs at and $u_{\mathrm{p}}=0.8 \mathrm{~km} \mathrm{~s}^{-1}$. Total energy is conserved for all cases. (b) The temporal evolution of the potential energy of the mesoparticles shows that the mesoparticle energy decreases as the internal specific heat increases.

\section{Results}

\subsection{Energetics and temperature during dynamical loading}

Figure 2(a) shows the temporal evolution of the total energy and that corresponding to the mesoparticles (see equation (4)) for simulations with $u_{\mathrm{p}}=0.8 \mathrm{~km} \mathrm{~s}^{-1}$ and various properties of the internal DoFs. In all cases the total energy (equation (3)) is conserved; this corresponds to mesoscale plus internal energies for the cases with $C^{\text {int }}=6 k_{\mathrm{B}}$ and $C^{\text {int }}=50 \mathrm{k}_{\mathrm{B}}$ and the mesoscale energy in the simulation with no coupling to internal DoFs (corresponding to regular MD and represented by a full line in figure 2). This shows that DID is applicable even under the extreme conditions and can describe decohesion, failure and evaporation of particles from solid materials; note that the internal energy of a DID mesoparticle in free flight remains constant. The mesoscale energy of the shocked material (see dashed and dotted lines in figure 2(a)) decreases as the internal specific heat is increased since the internal DoFs absorb more energy from the mesoparticles. This can also be seen in figure $2(b)$ where we plot the temporal evolution of the potential energy of the mesoparticles for the three simulations in figure $1(a)$ that differ only in the internal specific heat of the mesoparticles. In all three simulations the 

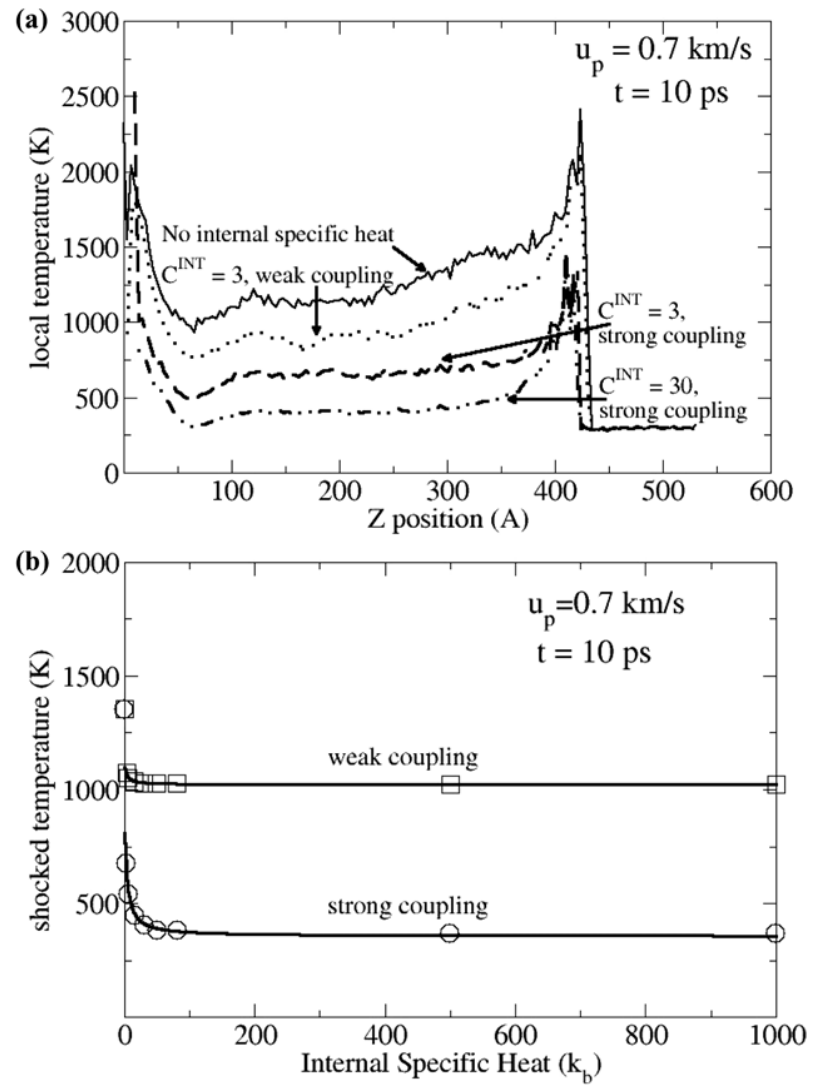

Figure 3. (a) Local molecular temperature $\left(T^{\text {meso }}\right)$ profiles along the loading direction at time $t=10 \mathrm{ps}$ for simulations corresponding to $u_{\mathrm{p}}=0.7 \mathrm{~km} \mathrm{~s}^{-1}$. We find that as the internal specific heat increases the temperature of the mesoparticles decreases. In addition, as the coupling constant becomes stronger, the mesoparticle temperature decreases as more energy can be exchanged in the short timescale involved in shock loading. $(b)$ Average molecular temperature of the shocked material as a function of the internal specific heat for $u_{\mathrm{p}}=0.7 \mathrm{~km} \mathrm{~s}^{-1}$ and two coupling constants. Symbols represent MD data and lines are fits to equation (7).

potential energy increases as the two compressive waves propagate into the target and projectile until, at time $t \sim 7 \mathrm{ps}$, the shock waves reach the free surface of the projectile. At this point a rarefaction fan propagates back into the material while the shockwave in the target continues its propagation leading to a rather constant value of the potential energy. At time $t \sim 14 \mathrm{ps}$ the second rarefaction starts propagating inward from the free surface of the projectile and the potential energy decreases as the two tensile waves propagate into the material. This expansion process can cause the failure of the material as shown in figure 1.

Figure 3(a) shows local molecular temperature $\left(T^{\text {meso }}\right)$ profiles along the loading direction at time $t=10 \mathrm{ps}$ for simulations corresponding to $u_{\mathrm{p}}=0.7 \mathrm{~km} \mathrm{~s}^{-1}$. We show results corresponding to four different conditions: (i) regular MD (no coupling to internal DoFs), (ii) $C^{\text {int }}=3 k_{\mathrm{B}}$ with strong coupling between internal and mesoscale DoFs $\left(\gamma=0.042 \mathrm{ps}^{-1}\right)$, (iii) $C^{\text {int }}=3 k_{\mathrm{B}}$ with weak coupling $\left(\gamma=0.0042 \mathrm{ps}^{-1}\right)$ and (iv) $C^{\text {int }}=30 k_{\mathrm{B}}$ and strong coupling. The shock wave initially heats up the molecular (explicit) DoFs and the energy cascades down to the high-frequency intra-molecular modes (described implicitly) increasing their temperature until they equilibrate with the molecular ones, i.e. the temperature of the 
two set of DoFs is the same. Figure 3(a) shows the temperature rise caused by the shock wave for various properties of the internal DoFs; the shock fronts can be seen in the projectile region between positions 400 and $450 \AA$ separating the unshocked material (with temperature $T=300 \mathrm{~K}$ ) from the shocked region. As expected the temperature of the shocked material decreases with increasing internal specific heat since the energy deposited in the material by the shock is shared between a larger number of DoFs. The strength of the coupling between the mesoparticles and internal DoFs also has an effect on the shock temperature. As the coupling decreases it takes a longer time for the internal and explicit DoFs to equilibrate and, due to the short timescales involved in shock loading, the internal and molecular temperatures do not reach equilibrium and, consequently, the molecular temperature increases. Figure 3(b) shows the average molecular temperature of the shocked material for shocks corresponding to $u_{\mathrm{p}}=0.7 \mathrm{~km} \mathrm{~s}^{-1}$ (averaging the temperature profiles in a region about $200 \AA$ long at time $10 \mathrm{ps}$ ) as a function of the specific heat per particle (approximated as $C^{\mathrm{int}}+3 k_{\mathrm{B}}$ ). The decrease in temperature as a function of specific heat observed in the simulations can be described with the following equation:

$$
T_{\text {shock }}=\frac{E_{\text {ini }}^{\text {meso }}+T_{\text {ini }}^{\text {int }} C^{\text {int }}}{3 k_{\mathrm{B}}+C^{\text {int }}},
$$

where $E_{\text {ini }}^{\text {meso }}$ is the initial energy of the mesoparticles (including the impact velocity) and $T_{\text {ini }}^{\text {int }}$ is the initial internal temperature of the mesoparticles. The numerator in equation (7) represents the total energy per particle and the denominator is an approximation to the specific heat per particle where the contribution of the mesoparticles is described by the classical harmonic value $\left(3 k_{\mathrm{B}}\right.$ per DOF). Note that for $C^{\mathrm{int}} \rightarrow \infty$ the shock temperature tends to the initial internal temperature $T_{\text {ini }}^{\text {int }}$, this behavior is only expected if the internal and molecular DoFs have time to fully equilibrate, i.e. very strong coupling or samples longer along the load direction such that that a longer time elapses before failure. Our simulations show that for finite couplings the shocked temperature in the $C^{\text {int }} \rightarrow \infty$ limit decreases as the coupling between the internal and molecular modes increases. Thus, we fit our MD results to equation (7) taking $E_{\mathrm{ini}}^{\text {meso }}$ and $T_{\text {ini }}^{\text {int }}$ as free variables; the resulting functions are shown as lines in figure $3(b)$. The data point corresponding to an internal specific heat of zero is not included in the fits. For the weak coupling we find $E_{\text {ini }}^{\text {meso }}=3361 K k_{\mathrm{B}}$ and $T_{\mathrm{ini}}^{\mathrm{int}}=1022 \mathrm{~K}$ and for the strong coupling we find $E_{\text {ini }}^{\text {meso }}=2884 K k_{\mathrm{B}}$ and $T_{\text {ini }}^{\text {int }}=356 K($ figure $3 b)$.

\subsection{Plastic deformation}

A planar shock front causes uniaxial compression in the material and the resulting deviatoric (non-hydrostatic) stress state acts as the driving force for plastic deformation. Figure 4 shows the temporal evolution of the stress along the shock direction for two different piston velocities and various properties of the internal DoFs. Consequently for strong enough shocks (above the so-called Hugoniot elastic limit (HEL)) plastic deformation occurs to relax the uniaxial compression toward a more hydrostatic state of lower free energy. In order to identify whether plastic deformation occurs in a simulation we perform an analysis based on the relative displacement of pairs of nearest neighbors, as mentioned in section 2 and described in more detail in [8]. Figure 5 shows MD snapshots at various times for simulations corresponding to $u_{\mathrm{p}}=0.75 \mathrm{~km} \mathrm{~s}^{-1}$ and (i) no internal DoFs (left panels), (ii) $C^{\text {int }}=3 k_{\mathrm{B}}$ and with weak coupling to the mesoparticles (center panels) and (iii) $C^{\text {int }}=3 k_{\mathrm{B}}$ and with strong coupling to the mesoparticles (panels on the right). Molecules are colored according to the results obtained from the plasticity analysis described above: dark gray molecules have undergone purely elastic deformation or no deformation at all (unshocked), black molecules have undergone $1 / 6\langle 112\rangle$ 

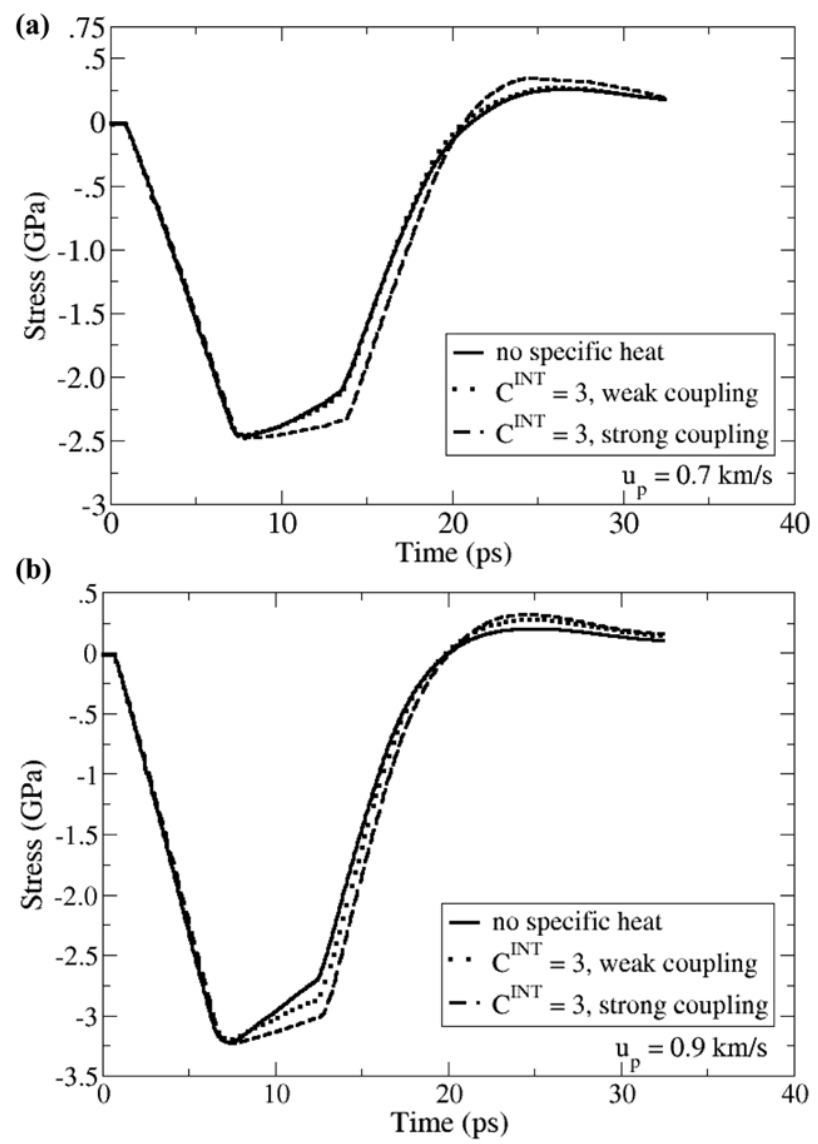

Figure 4. Temporal evolution of the stress along the shock direction for $u_{\mathrm{p}}=0.7 \mathrm{~km} \mathrm{~s}^{-1}(a)$ and $0.9 \mathrm{~km} \mathrm{~s}^{-1}(b)$. The initial decrease corresponds to the propagation of the two tensile waves, an abrupt change in slope occurs when the shock on the projectile reaches the free surface and transforms into a tensile wave; a second change in slope occurs when the shock wave on the target reaches the free surface. A fast increase in stress is observed as the two tensile waves travel back into the material leading eventually to a high tensile stress that can lead to failure. Note that the properties of the internal DOFs have little effect on the propagation of the compressive waves.

slip, i.e. black denotes the traces of partial dislocations with Burgers vector $1 / 6\langle 112\rangle$; light gray molecules have undergone a $1 / 2\left\langle\begin{array}{lll}1 & 0 & 0\end{array}\right.$ slip and are associated with full slip. Figure 5 shows that when no internal DoFs are present and the temperature rise is larger, plastic deformation nucleates during compression for shocks with $u_{\mathrm{p}}=0.75 \mathrm{~km} \mathrm{~s}^{-1}$ (figures $5(b)$ and $(c)$ ); some of this plastic deformation is reversed during expansion as some dislocations are able to trace back their steps when the sign of the stress changes, see figure $5(d)$; the final snapshot (figure 5(e)) shows the onset of failure. When the thermal role of internal DoFs is considered the temperature rise caused by the shockwave decreases with increasing $C^{\text {int }}$ and this has an effect on the ability of dislocations (the carriers of plastic deformation) to nucleate in the perfect crystal. When $C^{\text {int }}$ is increased to $3 k_{\mathrm{B}}$ and a weak coupling constant is used (figures $5(f)-(j)$ ) we observe plastic deformation although the density of dislocations is smaller than in the case with no internal DoFs. However $C^{\text {int }}=3 k_{\mathrm{B}}$ and a strong coupling constant (figures $5(k)-(o)$ ) leads to a very different behavior; under these conditions we observe no plastic deformation at all. This shows that the HEL depends not just on the inter-molecular 


\section{no internal specific heat}

(a) $t=2.5 \mathrm{ps}$

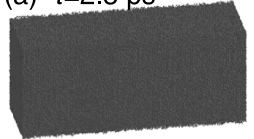

(b) $t=7.5 \mathrm{ps}$

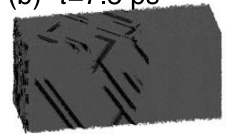

(c) $t=15 \mathrm{ps}$

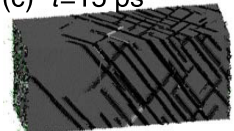

(d) $t=22.5 \mathrm{ps}$

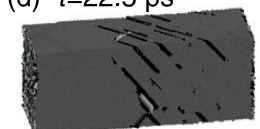

(e) $t=27.5 \mathrm{ps}$

$$
u_{P}=0.75 \mathrm{~km} / \mathrm{s}
$$

$$
\begin{gathered}
C^{I N T}=3 \\
\text { weak coupling }
\end{gathered}
$$

(f) $t=2.5 \mathrm{ps}$

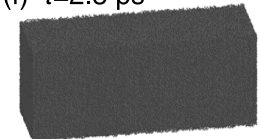

(g) $t=7.5 \mathrm{ps}$
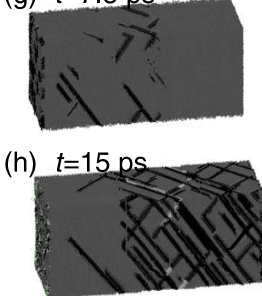

(i) $t=22.5 \mathrm{ps}$

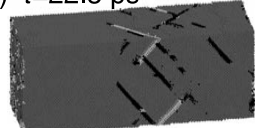

(j) $t=27.5 \mathrm{ps}$
$C^{I N T}=3$

strong coupling

(k) $t=2.5 \mathrm{ps}$

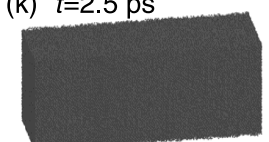

(I) $t=7.5 \mathrm{ps}$

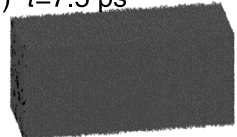

(m) $t=15 \mathrm{ps}$

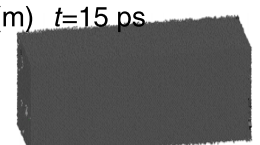

(n) $t=22.5 \mathrm{ps}$

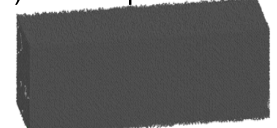

(o) $t=27.5 \mathrm{ps}$
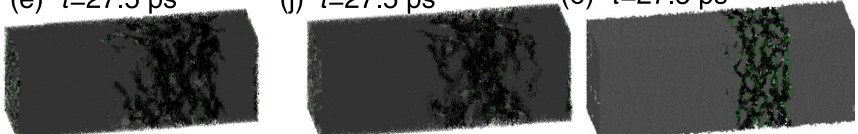

Figure 5. MD snapshots at various times for simulations corresponding to $u_{\mathrm{p}}=0.75 \mathrm{~km} \mathrm{~s}^{-1}$ and no internal DoFs (left panels), $C^{\text {int }}=3 k_{\mathrm{B}}$ with weak coupling (middle panel) and $C^{\text {int }}=3 k_{\mathrm{B}}$ with strong coupling (right panels). Dark gray spheres represent molecules that have undergone elastic or no deformation; molecules that have undergone $1 / 6\left\langle\begin{array}{lll}1 & 12 & 2\rangle\end{array}\right.$ slip (the traces of partial dislocations are represented as black spheres and white spheres denote molecules that have undergone a $1 / 2\langle 110\rangle$ slip (full dislocation).

DoFs but also on the properties of the intra-molecular ones, in particular their specific heat and how strongly they couple to molecular modes.

Figure 6 shows a map of the response of the material as a function of piston velocity and the specific heat of the internal DoFs for strong coupling. Each data point in figure 6 corresponds to a DID simulation; empty squares denote cases where failure did not occur and plasticity was not observed, triangles denote conditions that led to dynamical failure and did not show plasticity under compression, finally circles indicate cases where plasticity is observed during compression and the material fails. The solid line represents the HEL of the material for the conditions studied here (the HEL also depends on the size, microstructure and initial temperature) where the dependence on the specific heat of the internal DoFs can be seen. The threshold for failure is more insensitive to the properties of the internal DoFs but for $u_{\mathrm{p}}=0.2875 \mathrm{~km} \mathrm{~s}^{-1}$ we observe failure in a simulation with no internal DoFs and the material does not fail for $C^{\text {int }}=50 k_{\mathrm{B}}$.

\section{Discussion}

In this paper we used the DID method to characterize how the thermal properties of intra-molecular DoFs affect the nucleation of plastic deformation during shock loading 


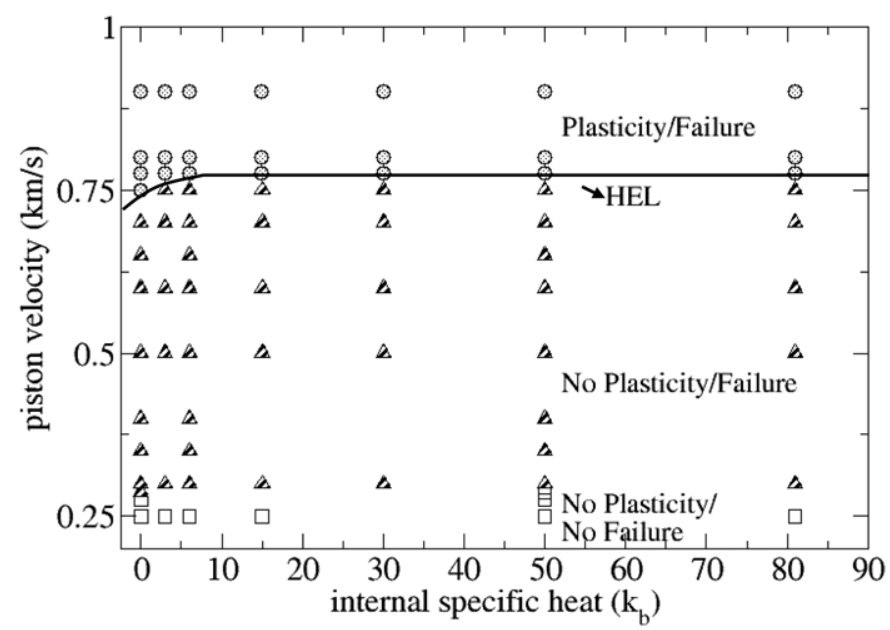

Figure 6. Map showing material response as a function of piston velocity and specific degrees of the internal DOFs for strong coupling. Squares represent purely elastic response; triangles represent cases where failure occurs but no plasticity is observed on compression; circles denote conditions where the material also exhibited plastic deformation. The solid line shows how the HEL depends on the internal specific heat.

and spallation in a model fcc molecular crystal. We kept our model very simple as opposed to trying to accurately describe a specific material since our goal is to characterize generally applicable phenomena; however DID can be used to describe specific materials with accuracy.

The first step in the development of an accurate, materials specific, coarse grain model is the development of a mesopotential that describes the interactions between the mesoparticles; this can be done using either experimental data or electronic structure calculations. As mentioned above, most molecular materials will require potentials that go beyond the twobody approximation we use in this paper. Beyond the mesopotential, a DID model requires additional information to describe the thermal behavior of the implicit DoFs, as can be seen in equations (1); these additional parameters can be calculated from first principles in a rather straightforward way.

(i) A key parameter in DID is the specific heat associated with the internal DoFs $\left(C^{\text {int }}\right)$. $C^{\text {int }}$ can be obtained from first principles using all atom MD simulations (with either a force field or ab initio electronic structure calculations). As discussed above, a direct calculation of specific heat from MD has the limitations of classical statistical mechanics. However, quantum effects can be incorporated using MD to obtain the vibrational density of states [22] and then computing the specific heat using quantum statistical mechanics and the density of states from MD [22]. This procedure essentially repopulates each mode taking into account quantum effects and provides an accurate description of equilibrium thermodynamic quantities.

(ii) The parameter $v$ in equations (1) represents the coupling between implicit DoFs and mesoparticles; i.e. how fast energy is transferred between the two sets of DoFs. This parameter can be obtained from classical or quantum all-atom MD simulations or from experiments. In this paper we studied two values of $v$ to explore its role in the dynamical response of our model material; a comparison of the temperature profiles shown in figure 3(a) with those resulting from non-equilibrium all-atom MD simulations [8] 
indicates that an accurate description for HMX would require a coupling constant similar to that in the weak coupling regime explored here.

(iii) Finally, the mean square vibrational frequency $\left\langle\omega^{2}\right\rangle$ in equations (1) is not a free parameter but depends on the mesopotential. $\left\langle\omega^{2}\right\rangle$ can be calculated from the vibrational density of states which in turn can be obtained from the velocity autocorrelation function [22].

We made other simplifying assumptions in this paper. We take the specific heat of the internal DoFs to be independent of temperature and pressure; this is not the case in real materials and DID can also be applied in such cases. We further assumed that the state of the internal DoFs can be fully described with a single state variable (the internal temperature); however, the internal DoFs can undergo structural processes such as structural transitions or chemical reactions whose description will require additional internal variables and generalizing the equations of motion. Chemical reactions have been incorporated in a mesoscale description recently by Maillet and Stoltz [23].

\section{Conclusions}

Our results show that the DID method can be used to describe thermo-mechanical processes at extreme conditions including processes involving failure and vaporization. We characterize how the specific heat of the internal DoFs and the strength of their coupling constant with molecular DOFs impact the thermo-mechanical behavior of molecular crystals. As the internal DoFs absorb more energy from the mesoparticles (either through an increase in internal specific heat or faster exchange of energy) the temperature rise caused by the dynamical mechanical load decreases influencing the thermo-mechanical response of the material. A decrease in molecular temperature makes the nucleation of dislocations more difficult and consequently the HEL is pushed to higher piston velocities, i.e. the HEL is affected by intra-molecular DOFs and their coupling with inter-molecular ones. We find that the dependence of the HEL piston velocity on internal specific heat is stronger for small molecules and can amount to about $3 \%$ for our fcc model crystals. We expect the dependence on internal properties to be more pronounced in most molecular crystals where open crystal structures lead to plastic deformation that exhibits a much stronger temperature dependence than in closed-packed fcc lattices.

The results of this paper have implications regarding the application of all-atom MD simulations to molecular materials containing high-frequency DoFs that are not accurately described by Newton's equations of motion; for such materials classical mechanics and statistical mechanics lead to an overestimation of the specific heat. This overestimation will lead to an underestimation of the temperature of the shock material and, likely, an overestimation of the HEL. Fortunately, quantum effects on thermal properties of intramolecular DoFs can be incorporated within the DID method (via a temperature dependent specific heat) in mesoscale simulations. Consequently, coarse graining can provide not only a computational less intensive alternative to all-atom MD but also a more accurate description of thermal processes; one should keep in mind, though, that the overall accuracy of either simulation is also strongly affected by the accuracy of the inter-atomic or meso-potentials.

\section{References}

[1] Gupta A, Peck G E, Miller R W and Morris K R 2005 J. Pharm. Sci. 94 2301-13

[2] Hooks D E, Ramos K J and Martinez R A 2006 J. Appl. Phys. 100024908

[3] Holian B L and Lomdahl P S 1998 Science 2802085

[4] Ismail-Beigi S and Arias T A 2000 Phys. Rev. Lett. 841499 
[5] Cawkwell M J, Nguyen-Manh D, Woodward C, Pettifor D G and Vitek V 2005 Science 3091059

[6] Schiøtz J and Jacobsen K W 2003 Science 3011357

[7] Strachan A, van Duin A C T, Chakraborty D, Dasgupta S and Goddard W A III 2003 Phys. Rev. Lett. 91098301

[8] Jaramillo E, Sewell T D and Strachan A 2007 Phys. Rev. B 76064112

[9] Voth G A 2002 Theoretical Methods in Condensed Phase Chemistry vol 5, ed S D Schawrtz (Dordrecht: Kluwer) p 47

[10] Strachan A and Holian B L 2005 Phys. Rev. Lett. 94014301

[11] Kalantar D H et al 2005 Phys. Rev. Lett. 95075502

[12] Loveridge-Smith A et al 2001 Phys. Rev. Lett. 862349

[13] McGrane S D, Moore D S and Funk D J 2007 J. Phys. Chem. A 108 9342-7

[14] Ashkenazy A and Averback R S 2005 Appl. Phys. Lett. 86051907

[15] Zhang C, Kalia R K, Nakano A and Vashishta P 2007 Appl. Phys. Lett. 91121911

[16] Muller-Plathe F 2002 Chem. Phys. 3754

[17] Fremer K 2003 Macromol. Chem. Phys. 204257

[18] Holian B L 2003 Europhys Lett. 64330

[19] Molinero V and Goddard W A III 2004 J. Phys. Chem. B 1081414

[20] Strachan A, Cagin T and Goddard W III 2001 Phys. Rev. B 63060103

[21] Srinivasan S G, Baskes M I and Wagner G J 2007 J. Appl. Phys. 101043504

[22] Berens P H, Mackay D H J, White G M and Wilson K R 1983 J. Chem. Phys. 792375

[23] Maillet J B and Stoltz G 2007 Europhys. Lett. 7868001 